\title{
0 Programa de Aquisição de Alimentos (PAA) como estratégia de enfrentamento aos desafios da COVID-19
}

\author{
Regina Helena Rosa Sambuichi 1 \\ Ana Flávia Cordeiro Souza de Almeida 1 \\ Gabriela Perin 1 \\ Paulo Asafe Campos Spínola 1 \\ Antônio Fernando Costa Pella 1
}

${ }^{1}$ Instituto de Pesquisa Econômica Aplicada / Diretoria de Estudos e Políticas Regionais, Urbanas e Ambientais, Brasília / DF - Brasil

\begin{abstract}
Este artigo analisou o Programa de Aquisição de Alimentos (PAA) como ferramenta para mitigar os impactos econômicos e sociais da COVID-19 no Brasil. Para isso, foram realizadas projeções de investimentos e analisadas as condições de operacionalização do programa no momento atual. Foi adotada uma metodologia quanti-quali de análise. $\mathrm{Na}$ abordagem quantitativa, foram construídos cenários com projeções de investimentos na política pública; na qualitativa, realizaram-se entrevistas não estruturadas com atores-chave na execução do programa. Os resultados revelaram que o aumento de recursos investidos no programa em um cenário otimizado pode proporcionar ampla capilarização dos benefícios. Identificou-se que o PAA é estratégico para o enfrentamento aos efeitos negativos da COVID-19 porque escoa os produtos da agricultura familiar, o que promove estruturação de cadeias produtivas, acesso a novos mercados e indução ao dinamismo econômico, além de atuar no combate à fome e à miséria de famílias que vivem em situação de vulnerabilidade. O programa não só apresenta condições de operacionalização durante a pandemia da COVID-19, como tem se adaptado à realidade atual, seguindo protocolos estabelecidos pelas autoridades de saúde. Concluiu-se que o PAA pode minimizar as crises sanitária e econômica, principalmente para a população mais vulnerável, apresentando ações que vão ao encontro das demandas da sociedade civil organizada e de recomendações de organismos nacionais e internacionais especializados, visando à redução dos impactos econômicos e sociais da pandemia causada pelo novo coronavírus.
\end{abstract}

Palavras-chave: COVID-19; segurança alimentar e nutricional; população vulnerável; impacto econômico; Programa de Aquisição de Alimentos.

\section{El Programa de Adquisición de Alimentos (PAA) como estrategia para enfrentar los desafíos de la COVID-19}

Este artículo analizó el Programa de Adquisición de Alimentos (PAA) como una herramienta para mitigar los impactos económicos y sociales de la COVID-19 en Brasil. Con este fin, se hicieron proyecciones de inversión y se analizaron las condiciones para operativizar el programa en el momento actual. Se adoptó una metodología de análisis cuanti-cualitativo. En el enfoque cuantitativo, los escenarios se construyeron con proyecciones de inversión en políticas públicas; en el cualitativo, se realizaron entrevistas no estructuradas con actores clave en la ejecución del programa. Los resultados revelaron que el aumento de recursos invertidos en el programa en un escenario optimizado puede proporcionar una amplia capilarización de los beneficios. Se identificó que el PAA es estratégico para enfrentar los efectos negativos de la COVID-19 porque incentiva la venta de los productos de la agricultura familiar, lo que promueve la estructuración de las cadenas de producción, el acceso a nuevos mercados e induce al dinamismo económico, además de actuar en la lucha contra el hambre y la miseria de las familias que viven en situación de vulnerabilidad. El programa no solo presenta condiciones operativas durante la pandemia de COVID-19, sino que también se ha adaptado a la realidad actual, siguiendo los protocolos establecidos por las autoridades sanitarias. Se concluyó que el PAA puede minimizar las crisis sanitaria y económica, especialmente para la población más vulnerable, presentando acciones que satisfagan las demandas de la sociedad civil organizada y las recomendaciones de organizaciones nacionales e internacionales especializadas destinadas a reducir los impactos económicos y sociales de la pandemia causada por el nuevo coronavirus.

Palabras clave: COVID-19; seguridad alimentaria y nutricional; población más vulnerable; Programa de Aquisição de Alimentos. 


\section{The Food Acquisition Program (PAA) as a strategy to face the challenges of COVID-19}

This article analyzed the Food Acquisition Program (PAA) as a tool to mitigate the economic and social impacts of COVID-19 in Brazil. The study conducted investment projections and analyzed the conditions to operate the program during the pandemic, adopting quantitative-qualitative analysis. As for the quantitative approach, the study worked with projections of public policy investments; the qualitative policy was carried out with unstructured interviews with key actors. The results showed that, in an optimal scenario, the rise of investment could provide a wide capillarization of the benefits of the program. The results point out that the PAA is strategic to face the negative effects of COVID-19 because it helps family farming products reach the consumers, structuring production chains, facilitating access to new markets, and inducing economic dynamics, in addition to combating hunger and extreme poverty of families living in vulnerability. Also, the research found that the program is in operational conditions and adapted to the reality of the pandemic, following protocols established by health authorities. Therefore, the PAA can minimize health and economic crises for the most vulnerable population. It addresses the demands of the organized civil society and recommendations from national and international organizations specialized in reducing the economic and social impacts of the COVID-19 pandemic.

Keywords: COVID-19; food and nutrition security; vulnerable populations; economic impact; Programa de Aquisição de Alimentos.

\section{INTRODUÇÃO}

Diante da pandemia provocada pelo novo coronavírus, diversos países e organizações do mundo têm anunciado medidas com o objetivo de amenizar os impactos negativos na economia e atenuar danos aos grupos que vivem em condições sociais mais vulneráveis.

A paralisação econômica e social provocada pelo isolamento apresenta como consequências fechamento de restaurantes, suspensão de aulas em escolas públicas e privadas, enfraquecimento das atividades de trabalhadores informais, aumento de demissões e redução na disponibilidade de alimentos distribuídos a pessoas que vivem em situação de risco alimentar. Tais fatos poderão ter efeitos diretos no aumento da vulnerabilidade social e da insegurança alimentar e nutricional (Insan).

No caso da agricultura familiar, os efeitos da crise sanitária e as medidas necessárias de prevenção geram maior dificuldade de comercialização, pois restaurantes populares, escolas públicas e feiras são parte importante do destino da produção familiar. Além da redução de funcionamento desses estabelecimentos, há ainda a preocupação com as questões de logística, já que houve interrupção de parte dos serviços de transportes público e privado, os quais ligam o meio rural ao urbano.

As consequências da redução dos canais de comercialização e das dificuldades de logística são altamente danosas à sociedade como um todo. Em primeiro lugar, porque repercutem na redução da renda dos agricultores familiares e do acesso à alimentação adequada para a população em situação de vulnerabilidade social (Valadares et al., 2020). Em segundo lugar, porque a circunstância poderá aumentar o consumo de alimentos ultraprocessados, com baixo valor nutricional (Oliveira, Abranches, \& Lana, 2020). Em terceiro lugar, sem conseguir escoar a produção, há o risco de perda dos alimentos na propriedade, enquanto um número crescente de pessoas passa fome nas cidades. Por fim, em médio prazo, tem-se a ameaça de retração da oferta, aumentando o risco de desabastecimento após a pandemia e, consequentemente, a elevação dos preços de produtos do gênero alimentício (Valadares et al., 2020).

É um cenário preocupante que se agrava face ao enfraquecimento das políticas de proteção social, em especial aquelas voltadas ao desenvolvimento rural sustentável em nível federal, o que vem ocorrendo há alguns anos devido à crise fiscal e à política de austeridade adotadas pelo governo brasileiro. É nesse contexto que o Programa de Aquisição de Alimentos (PAA) se insere. Criado pela Lei $\mathrm{n}^{\circ} 10.696$, de 2 de julho de 2003, e alterada pela Lei $\mathrm{n}^{\circ} 12.512$, de 14 de outubro de 2011, é uma 
política pública que apresenta como focos centrais o incentivo à agricultura familiar e o combate à insegurança alimentar e nutricional. Por esse motivo, trata-se de um instrumento com potencial para ser usado pela administração pública no enfrentamento aos possíveis danos econômicos e sociais provocados pela COVID-19.

Inserindo-se no contexto da Política Nacional de Segurança Alimentar e Nutricional, o PAA tem, entre seus objetivos principais, promover a inclusão produtiva dos agricultores mais pobres e garantir à população o acesso à alimentação saudável. Ele apresenta como beneficiários diretos os agricultores familiares (beneficiários fornecedores) e as pessoas em situação de risco alimentar (beneficiários consumidores), atuando por meio de diversas modalidades e formas de implementação.

O programa opera atualmente em seis modalidades: compra com doação simultânea (CDS), compra direta (CDAF), apoio à formação de estoques, incentivo à produção e ao consumo de leite, compra institucional e aquisição de sementes. A principal delas é a CDS, em que o programa compra os alimentos produzidos pelos agricultores familiares e os doa a entidades da rede socioassistencial, equipamentos públicos de segurança alimentar e nutricional - como restaurantes populares e bancos de alimentos - para a rede pública e/ou filantrópica de ensino, hospitais e outros, os quais, por sua vez, fazem a distribuição às pessoas que precisam desses produtos.

Além de diferentes modalidades, o programa apresenta também diferentes operadores e fontes de recursos. Entre os operadores estão a Companhia Nacional de Abastecimento (Conab), além de estados, Distrito Federal e municípios. Os recursos são provenientes sobretudo do Ministério da Cidadania, mas podem vir também do Ministério da Agricultura, da Pecuária e do Abastecimento (Mapa), como no caso da modalidade formação de estoques, ou do ente público da administração direta ou indireta interessado na compra, no caso da modalidade compra institucional.

Por se tratar de uma política intersetorial e de natureza horizontalizada, acredita-se que o PAA seja um instrumento de alta capilaridade para o combate à crise sanitária provocada pela COVID-19, sendo uma medida tempestiva de efeito rápido, pois o programa já está criado e consolidado, com marcos regulatórios instituídos e uma estrutura de implementação ativa, além de ter benefícios no âmbito econômico e social comprovados em estudos de caso realizados em todas as regiões do Brasil.

Dessa maneira, este artigo tem como objetivo analisar o PAA como ferramenta para mitigar os impactos econômicos e sociais da COVID-19 no país. Para isso, foram realizadas projeções de investimentos e análises das condições de operacionalização do programa no momento atual. Iniciando por esta introdução, o artigo apresenta, na sequência, uma breve revisão de literatura sobre o PAA, seus benefícios e como ele se insere no contexto de pandemia. Em seguida, são apresentadas seções com aspectos metodológicos, resultados e discussões e, por fim, a conclusão do estudo.

\section{REVISÃO DE LITERATURA}

\subsection{Impactos da COVID-19 sobre a Produção de Alimentos e Estratégias de Enfrentamento}

A Organização das Nações Unidas para Alimentação e Agricultura (FAO), a Organização Mundial da Saúde (OMS) e a Organização Mundial do Comércio (OMC), por meio de uma declaração conjunta, orientaram que países devem pautar suas ações na diminuição de impactos negativos no suprimento de alimentos, conter a fragilização do comércio e promover segurança alimentar diante da pandemia causada pelo novo coronavírus (FAO, 2020). 
Segundo a FAO (2019), sem uma situação pandêmica, o mundo já lida com aproximadamente 820 milhões de pessoas em situação de fome crônica e 113 milhões em Insan aguda. Esse público se encontra em situação mais vulnerável e pode perder o acesso ao alimento em decorrência da COVID-19, portanto deve ser o foco para a construção de medidas emergenciais.

Os esforços para combater os efeitos negativos da pandemia do novo coronavírus têm se concentrado, em muitos países, em evitar (ou reduzir) interrupções que influenciam a força de trabalho e impactam a produção de alimentos e a geração de emprego e renda. Dessa forma, as políticas públicas de resposta à COVID-19 devem ser implementadas de modo a garantir a produção doméstica e a renda do agricultor. Essas medidas funcionam como amortecedores no pós-pandemia, responsáveis por manter "níveis adequados de produção doméstica e renda dos agricultores usados para apoiar a economia e garantir a segurança alimentar" (FAO, 2020a, p. 2).

No Brasil, uma parcela da sociedade já vive em condições de insegurança alimentar e nutricional, e a nova dinâmica que a pandemia tem provocado tende a agravar o problema. Baseando-se em experiências recentes, para evitar a severidade das crises alimentares durante e após a crise sanitária, são necessárias ações preventivas de combate à Insan, como ampliação de programas de proteção social, atendimento das necessidades alimentares imediatas de pessoas em situação vulnerável e redução dos custos relacionados ao comércio (FAO, 2020b).

A Confederação Nacional da Agricultura e Pecuária (CNA), por meio de Boletim Informativo, destacou que o fechamento de parte do comércio e a consequente redução da demanda e alta volatilidade de preços têm afetado negativamente vários setores da agropecuária, indicando o aumento das compras governamentais de alimentos como uma das medidas necessárias para minimizar esses danos (CNA, 2020).

Organizações da sociedade civil, bem como movimentos sindicais e sociais, também se manifestaram, apresentando demandas e formulando documentos que foram aderidos por 140 entidades civis. Os documentos apontam as providências emergenciais a serem implementadas durante e após a pandemia - entre elas, ampliação de demanda dos produtos da agricultura familiar por meio de mercados institucionais, ações de promoção da geração de renda, segurança financeira e produtiva da agricultura familiar, além de regulação do sistema alimentar e preços agrícolas (Valadares et al., 2020).

Uma pesquisa realizada pelo Instituto Conexões Sustentáveis, pela União Nacional das Cooperativas de Agricultura Familiar e Economia Solidária (Unicafes) e pelo Conselho Nacional de Populações Extrativistas (CNS) identificou que 80\% dos participantes de negócios comunitários, como cooperativas e associações, que participaram do estudo não terão saúde financeira para dar continuidade às atividades após junho de 2020 (Pontes, 2020).

A redução da demanda de consumo em restaurantes, a paralisação de comércio e escolas, a interrupção temporária de entregas, a fragmentação da estrutura de logística, a dificuldade de comunicação entre o meio urbano e as áreas mais afastadas já são percebidas em nível local como consequências da COVID-19 (Instituto Conexões Sustentáveis [Conexsus], 2020).

Outras rupturas fortes que ocorreram na comercialização da agricultura familiar foram a interrupção temporária das feiras livres e a paralisação das compras do Programa Nacional de Alimentação Escolar (PNAE) em muitos estados e municípios do Brasil. A primeira é o principal canal de comercialização da agricultura familiar, o que em médio prazo pode comprometer não só as cadeias produtivas, mas também a segurança alimentar e nutricional (SAN) e a economia em 
nível local. A segunda prevê a compra direta de $30 \%$ do valor repassado em produtos da agricultura familiar, com base na Lei ${ }^{\circ}$ 11.947, de 2009. Todos esses aspectos refletem na diminuição da renda de pequenos produtores e enfraquecem a cadeia produtiva e a economia local, provocando perda de produção ou insegurança quanto ao planejamento do plantio e à aquisição de insumos para as safras de inverno, além do aumento no número de pessoas em situação de vulnerabilidade social e insegurança alimentar (ANA, 2020; Conexsus, 2020).

A fim de minimizar esses danos, na esfera privada têm ocorrido iniciativas muito importantes. Junto com a empresa de assistência técnica e extensão rural do estado do Rio de Janeiro, a Fundação Banco do Brasil (FBB) firmou um termo de compromisso com cooperativas e associações de agricultores familiares que define que a FBB fará aquisição de produtos da agricultura familiar de quatro municípios do Rio de Janeiro. "Os alimentos chegarão aos trabalhadores informais, que no momento estão sem a possibilidade de gerar renda, identificados nas áreas rurais e urbanas das cidades da Região Serrana do Rio de Janeiro" (FBB, 2020).

Na comercialização direta, têm sido usadas estratégias e adequações para a entrega de produtos da agricultura familiar. Vem sendo recorrente a solicitação dos consumidores por meio de telefone, aplicativos ou redes sociais. Com isso, os agricultores montam cestas e fazem as entregas diretamente no domicílio do consumidor (Brasil, 2020). Contudo, os números negativos observados nos últimos levantamentos realizados indicam que essas alternativas de comercialização não têm sido suficientes para dar vazão aos produtos da agricultura familiar e evitar as perdas de alimentos (CNA, 2020; Pontes, 2020).

\subsection{Efeitos do PAA na Economia Local, na Estruturação das Cadeias Alimentares e na Promoção da Segurança Alimentar e Nutricional}

O PAA está entre os programas públicos de apoio à comercialização e à promoção de segurança alimentar mais estudados. Uma revisão sistemática de literatura realizada por Almeida, Perin, Policarpo e Sambuichi (2020) identificou análises empíricas que abordaram as dimensões econômicas, sociais e ambientais do PAA. No âmbito econômico, as autoras encontraram pesquisas as quais apontam que o PAA atua como uma ferramenta capaz de promover estruturação de cadeias produtivas, acesso a novos mercados e indução ao dinamismo econômico (Quadro 1).

QUADRO 1 NÚMERO DE EVIDÊNCIAS EMPÍRICAS DO PAA NA DIMENSÃO ECONÔMICA

\begin{tabular}{ccc} 
Dimensão & Elementos de análise & $\mathbf{N}^{\circ}$ de estudos \\
\hline Dinamização da economia local & 21 \\
\hline Econômica & Melhoria da qualidade de produtos & 23 \\
& Acesso a novos mercados & 15
\end{tabular}

Fonte: Adaptado de Almeida et al. (2020). 
Entre os efeitos positivos do programa, observou-se um aumento na quantidade de produtos vendidos pelos agricultores, com impactos positivos em receita média, lucro líquido, investimentos totais e tempo de retorno do capital das unidades produtivas (Santos, Soares, \& Benavides, 2015). Além disso, a garantia de comercialização proporcionada pelo PAA e o consequente aumento da renda dos agricultores familiares acabam influenciando a expansão do consumo dessas famílias, provocando um ciclo virtuoso de desenvolvimento na economia local (Agapto, Borsatto, Esquerdo, \& Bergamasco, 2012).

Além de dinamizar a economia local e atuar como indutor do consumo, o programa apresenta a característica importante de incentivar uma produção de alimentos mais pulverizada no território nacional e aproximar o produtor do consumidor final. Ao potencializar os circuitos curtos, que são de grande relevância para o nível local e regional, o PAA induz modificações na dinâmica interna da unidade familiar de produção, com reflexos na dinâmica de consumo, na diversificação e na sustentabilidade da atividade econômica (Rambo \& Denz, 2015).

Ao analisar o PAA pela óptica da economia e da comercialização, observa-se que, em momentos de perda momentânea dos canais de comércio, o programa assume o papel de escoar a produção em nível local, ao mesmo tempo que mantém o equilíbrio de preços (Agostini \& Bourscheidt, 2018).

No âmbito social, o trabalho de Almeida et al. (2020) identificou estudos que mostraram que o PAA proporciona aumento e diversificação dos salários e/ou da renda, já que é um canal de comercialização que possibilita a garantia da venda e melhora a qualidade da alimentação dos beneficiários fornecedor, que pratica o autoconsumo de alimentos produzidos, e consumidor, que passa a ter acesso aos alimentos diversificados do ponto de vista nutricional. Ademais, incentiva o consumo de alimentos de qualidade, proporcionando ao público-alvo a melhoria na saúde (Quadro 2).

\section{QUADRO 2 NÚMERO DE EVIDÊNCIAS EMPÍRICAS DO PAA NA DIMENSÃO SOCIAL}

\begin{tabular}{|c|c|c|}
\hline Dimensão & Elementos de análise & $\mathrm{N}^{0}$ de estudos \\
\hline \multirow{7}{*}{ Social } & Aumento e diversificação dos salários e/ou da renda & 70 \\
\hline & Comercialização/garantia de venda & 56 \\
\hline & $\begin{array}{l}\text { Autoconsumo e qualidade da } \\
\text { alimentação de agricultores }\end{array}$ & 22 \\
\hline & $\begin{array}{l}\text { Melhoria na qualidade da alimentação dos beneficiários } \\
\text { consumidores }\end{array}$ & 36 \\
\hline & Aumento do acesso & 15 \\
\hline & Incentivo ao consumo de alimento de qualidade & 7 \\
\hline & Melhoria na saúde & 11 \\
\hline
\end{tabular}

Fonte: Adaptado de Almeida et al. (2020). 
Sambuichi, Almeida, Perin, e Moura (2019a), ao analisar o perfil dos municípios que acessaram o PAA entre 2011 e 2016, identificaram que aqueles que apresentam um menor Produto Interno Bruto (PIB) per capita, menor índice de desenvolvimento humano municipal (IDHM), maior número de agricultores familiares e maior percentual de habitantes extremamente pobres apresentam probabilidade maior de acesso ao programa. Entre as regiões, observou-se uma probabilidade de maior acesso no Nordeste e no Norte, onde se concentram os maiores índices de pobreza rural. Esses resultados vão ao encontro das finalidades da política de promover a inclusão social dos agricultores mais pobres e atender às populações mais vulneráveis à Insan.

Do ponto de vista dos beneficiários consumidores, há aumento na diversificação do cardápio alimentar e melhoria na qualidade das refeições em escolas municipais e estaduais (Barth-Teixeira, Santos, Gregolin, \& Christoffoli, 2017). Além disso, a ampliação do acesso a alimentos diversificados influenciou positivamente os rendimentos escolares, a assiduidade e a redução dos índices "ruim" e "regular" de estudantes (Martins, 2013 como citado em Ortega, Jesus, \& Só, 2016), bem como a criação de novos e melhores hábitos alimentares (Salgado, Dias, Silva, Santos, \& Cunha, 2015).

No que tange aos beneficiários fornecedores, chama-se a atenção para o autoconsumo, pois a expansão da diversidade de produtos cultivados para comercialização trouxe melhorias na alimentação dos próprios agricultores, haja vista que as famílias produtoras incorporam à alimentação parte da produção e passam a realizar uma dieta alimentar mais estruturada (Chmiekewska, Souza, \& Lourete, 2010).

Dessa forma, diversos estudos vêm mostrando que o PAA tem a capacidade de promover acesso à alimentação e melhorar a qualidade e os hábitos alimentares das populações mais vulneráveis, qualificando-se como imprescindível para mitigar os efeitos da pandemia na população em risco social e alimentar no Brasil. A promoção do aumento do acesso aos alimentos in natura e minimamente processados se torna mais importante porque seu consumo, em especial quando se trata de crianças, idosos e pessoas com imunodepressão, fortalece o sistema imunológico e garante maior resistência às doenças. A esses benefícios nutricionais se agregam os impactos econômicos para as regiões onde o PAA está presente.

\section{ASPECTOS METODOLÓGICOS}

Para a elaboração deste artigo, utilizou-se abordagem quanti-quali de análise. No estudo quantitativo, foram analisadas compras e doações do PAA realizadas em anos anteriores, estimando valores totais aplicados, quantidades de alimentos adquiridos, número de beneficiários fornecedores, municípios abrangidos e atendimentos a beneficiários consumidores realizados. Com base nesses quantitativos, foram projetados os resultados que seriam obtidos em três diferentes cenários de investimentos no programa, buscando estimar os possíveis benefícios gerados em cada caso. Para isso, usaram-se microdados referentes a registros administrativos de compras e doações do PAA do período de 2011 a 2018, disponibilizados pelo Ministério da Cidadania e pela Conab para esta pesquisa, analisados por meio do software $\mathrm{R}$.

O cenário mais conservador (cenário 1) previu a aplicação total, sem contingenciamento, do valor de R 186 milhões previsto no orçamento, já incluindo as emendas parlamentares, para ser aplicado no programa no ano de 2020. O cenário intermediário (cenário 2) previu a aplicação total no PAA do valor de R 500 milhões previsto na Medida Provisória n 957, de 24 de abril de 2020, que abriu crédito extraordinário, em favor do Ministério da Cidadania, para enfrentar a emergência de saúde pública em razão da pandemia. O cenário otimizado (cenário 3) previu os resultados da aplicação 
de R 1 bilhão, valor demandado pelos movimentos sociais para ser aplicado no programa em 2020 (ANA, 2020).

Nos cenários 1 e 2, os quantitativos apresentados seguem as tendências observadas nos anos de 2011 a 2018 para cada valor total aplicado, sendo estimados por meio de regressão linear pelo método dos mínimos quadrados ordinários ([MQO], Greene, 2007). Os parâmetros das equações usadas para estimar os valores são apresentados na Tabela 1.

No cenário 3, as projeções de número de agricultores foram otimizadas visando atingir um maior número de beneficiários fornecedores. Para isso, o valor por agricultor foi fixado para um valor médio de $\mathrm{R} \$ 600,00$ mensais com o objetivo de atender a um número maior de agricultores, o que daria um total de $\mathrm{R} \$ 4.800,00$ por agricultor, considerando 8 meses no ano contados a partir de maio. Os demais quantitativos foram estimados pelo mesmo método adotado nos outros dois cenários.

\section{TABELA 1 PARÂMETROS DAS REGRESSÕES UTILIZADAS PARA ESTIMAR QUANTIDADE DE ALIMENTOS E NÚMERO DE AGRICULTORES, MUNICÍPIOS E ATENDIMENTOS APRESENTADOS NOS CENÁRIOS 1 E 2; TOTAL DE ALIMENTOS E NÚMERO DE MUNICÍPIOS E ATENDIMENTOS APRESENTADOS NO CENÁRIO 3}

\begin{tabular}{lcccc} 
Variáveis & Quantidade & Agricultores & Municípios & Atendimentos \\
\hline Intercepto & $-20,98652$ & $17,89329^{*}$ & $1785,686^{* *+*}$ & $6,441143^{*+* *}$ \\
& $(22,16934)$ & $(5,16181)$ & $(85,709)$ & $(1,066342)$ \\
Valor & $0,44142^{*+*+}$ & $0,1329^{* * *}$ & $1,581^{* *+*}$ & $0,00563^{* *}$ \\
& $(0,03104)$ & $(0,007228)$ & $(0,120)$ & $(0,001493)$
\end{tabular}

Nota: ${ }^{* * *} \mathrm{p}<0,01 ;{ }^{* *} \mathrm{p}<0,05 ;{ }^{*} \mathrm{p}<0,1$. Desvios-padrões entre parênteses.

Fonte: Elaborada pelos autores.

$\mathrm{Na}$ parte qualitativa, foram realizadas entrevistas não estruturadas com representantes do Ministério da Cidadania, da Conab e da Articulação Nacional de Agroecologia (ANA), visando coletar informações acerca da situação atual de implementação do PAA, da viabilidade de execução dos valores projetados nos diferentes cenários e das adaptações que têm sido realizadas na execução do programa em razão das necessidades geradas pela pandemia. A escolha por esses atores buscou abranger representantes do governo e da sociedade civil que atuam nessa temática e se baseou nos seguintes critérios: o Ministério da Cidadania é o principal gestor e fonte de recursos do PAA; a Conab é uma das principais executoras do programa; a ANA é um dos importantes espaços de articulação e convergência, reunindo representantes de movimentos, redes e organizações da sociedade civil engajados no fortalecimento da agricultura familiar. 


\section{RESULTADOS E DISCUSSÕES}

\subsection{Os Cenários de Operacionalização do PAA}

A Tabela 2 mostra valores monetários aplicados, quantidade de alimentos adquiridos e beneficiários abrangidos pelo programa no período de 2011 a 2018. Esses dados apresentam, em conjunto, todas as modalidades operadas com recursos do Governo Federal, provenientes do Ministério da Cidadania e do Mapa, incluindo também as doações da modalidade CDS. Os números, porém, não incluem as compras da modalidade compra institucional, a qual é operada com recursos próprios do ente da administração direta ou indireta que faz a aquisição dos alimentos e da qual não temos dados disponíveis sobre os recursos aplicados no período.

\section{TABELA 2 VALORES, QUANTIDADES ADQUIRIDAS E BENEFICIÁRIOS DO PAA' NO PERÍODO DE 2011 A 2018}

\begin{tabular}{|c|c|c|c|c|c|c|}
\hline Período & $\begin{array}{c}\text { Valor aplicado }{ }^{1} \\
\text { (R\$ milhões) }\end{array}$ & $\begin{array}{l}\text { Quantidades de } \\
\text { produtos } \\
\text { (Mil toneladas) }\end{array}$ & $\begin{array}{c}\mathrm{N}^{\circ} \text { de } \\
\text { agricultores } \\
\text { (Milhares) }\end{array}$ & $\begin{array}{c}\mathrm{N}^{0} \text { de } \\
\text { municípios } \\
\text { beneficiados }{ }^{2}\end{array}$ & $\begin{array}{c}\mathrm{N}^{\circ} \text { de entidades } \\
\text { beneficiadas } \\
\text { (Milhares) }\end{array}$ & $\begin{array}{c}\mathrm{N}^{\circ} \text { de atendimentos } \\
\text { de beneficiários } \\
\text { consumidores } \\
\text { (Milhões) }\end{array}$ \\
\hline 2011 & $1.006,8$ & 465,4 & 156,6 & 3429 & 25,0 & 12,7 \\
\hline 2012 & $1.157,1$ & 468,9 & 175,8 & 3630 & 23,4 & 12,8 \\
\hline 2013 & 588,6 & 253,9 & 93,4 & 2635 & 17,7 & 8,5 \\
\hline 2014 & 752,6 & 309,9 & 112,0 & 3065 & 14,2 & 9,4 \\
\hline 2015 & 668,6 & 239,3 & 96,7 & 2665 & 11,2 & 11,0 \\
\hline 2016 & 426,6 & 150,8 & 77,3 & 2428 & 11,3 & 10,7 \\
\hline 2017 & 362,6 & 143,6 & 68,2 & 2456 & 10,3 & 9,1 \\
\hline 2018 & 232,7 & 93,8 & 53,6 & 2192 & 11,1 & 6,5 \\
\hline Total ${ }^{3}$ & $5.195,6$ & $2.125,5$ & 454,8 & 4610 & 65,1 & 80,8 \\
\hline
\end{tabular}

${ }^{1}$ Valores corrigidos para dezembro de 2018 pelo IPCA-E.

${ }^{2}$ Inclui os municípios do agricultor que forneceu produtos para o PAA em pelo menos 1 modalidade no período e da entidade que recebeu doações da modalidade CDS.

${ }^{3}$ Os totais de beneficiários são apresentados para o total do período sem repetição.

Fonte: Elaborada pelos autores com base em microdados disponibilizados pelo Ministério da Cidadania.

Observa-se na Tabela 2 que os valores de compras foram mais elevados nos anos de 2011 e 2012, tiveram queda acentuada em 2013, uma recuperação parcial em 2014 e 2015, voltando a cair fortemente em anos mais recentes em virtude de cortes orçamentários causados pela crise econômica e pela redução de gastos do Governo Federal.

A redução dos valores aplicados foi acompanhada de uma pulverização maior dos gastos entre os municípios, já que a queda no número de municípios beneficiados não ocorreu na mesma proporção 
que a dos recursos aplicados no programa (Tabela 2). Com isso, os municípios passaram a receber valores médios mais baixos, o que pode ter afetado o potencial do programa de promover a dinamização da economia local. Apesar disso, o fato de o PAA ter continuado a operar em um número significativo de municípios indica que ele mantém a capilaridade. Portanto, se os montantes aplicados voltarem a crescer, o programa pode ter condições de voltar a atuar rapidamente com a abrangência que já teve em anos anteriores.

As projeções de alcance dos benefícios gerados pelo PAA nos diferentes cenários de investimento de recursos investigados nesta pesquisa são apresentadas na Tabela 3.

\section{TABELA 3 PROJEÇÕES DE ALCANCE DOS BENEFÍCIOS DO PAA EM DIFERENTES CENÁRIOS DE INVESTIMENTO DE RECURSOS}

\begin{tabular}{|c|c|c|c|c|c|c|}
\hline Cenários & $\begin{array}{l}\text { Valor (R\$ } \\
\text { milhões) }\end{array}$ & $\begin{array}{l}\text { Quantidades } \\
\text { adquiridas } \\
\text { (Mil toneladas) }\end{array}$ & $\begin{array}{l}\mathrm{N}^{\circ} \text { de } \\
\text { agricultores } \\
\text { beneficiados } \\
\text { (Milhares) }\end{array}$ & $\begin{array}{l}\mathrm{N}^{0} \text { de municípios } \\
\text { beneficiados }\end{array}$ & $\begin{array}{c}\mathrm{N}^{0} \text { de } \\
\text { atendimentos de } \\
\text { consumidores } \\
\text { (Milhões) }\end{array}$ & $\begin{array}{c}\text { Valor Médio } \\
\text { anual por } \\
\text { agricultor } \\
\text { (R\$) }\end{array}$ \\
\hline Cenário 1 & 186 & 61 & 42 & 2080 & 7,5 & $4.365,89$ \\
\hline Cenário 2 & 500 & 199 & 84 & 2576 & 9,3 & $5.928,64$ \\
\hline Cenário 3 & 1000 & 420 & 208 & 3366 & 12,0 & $4.800,00$ \\
\hline
\end{tabular}

Fonte: Elaborada pelos autores com base em microdados disponibilizados pelo Ministério da Cidadania.

Considerando-se o cenário 1, com o orçamento previsto de $\mathrm{R} \$ 186$ milhões, estima-se que seriam adquiridas 61 mil toneladas de alimentos, atendendo a cerca de 42 mil agricultores familiares, em 2.080 municípios, com 7,5 milhões de atendimentos a beneficiários consumidores. Os valores apresentados não correspondem ao número de beneficiários consumidores atendidos, mas à quantidade de atendimentos das entidades, sendo que a forma como são feitos varia de acordo com o tipo de entidade que recebe as doações.

No cenário 2, com o recurso de R 500 milhões previsto na MP 957, estima-se que seja possível adquirir cerca de 199 mil toneladas de alimentos, atendendo aproximadamente a 84 mil agricultores familiares, em 2.576 municípios e com 9,3 milhões de atendimentos a consumidores. Já no cenário 3, considerando um investimento de R 1 bilhão, valor próximo ao aplicado nos anos de maior atuação do programa, 2011 e 2012, a estimativa total seria adquirir 420 mil toneladas de alimentos, beneficiando 3.366 municípios, com 12 milhões de atendimentos a beneficiários consumidores. A otimização do recurso, limitando o valor por agricultor a $\mathrm{R} \$ 4.800,00$ nos 8 últimos meses de 2020, permitiria atingir uma quantidade maior de beneficiários fornecedores, beneficiando diretamente 208 mil agricultores familiares.

Esses resultados mostram que, sem investimento de recursos adicionais no programa (cenário 1), o PAA atenderia em 2020 ainda menos beneficiários do que em 2018 (apresentado na Tabela 2). 
Portanto, com base em todos os benefícios potenciais do programa, esse não seria um cenário recomendável, sobretudo diante das necessidades geradas pela atual crise econômica causada pela pandemia, o que reforça a necessidade do crédito extraordinário previsto na MP no 957.

Conforme nos mostram as projeções do cenário 3, todavia, se mais recursos forem disponibilizados para o programa, operando em um patamar de $\mathrm{R} \$ 1$ bilhão ou mais, ele poderá atender a um número considerável de beneficiários diretos, além do benefício indireto de estimular a dinamização das economias locais e as cadeias de produção de alimentos saudáveis, a fim de garantir o abastecimento e promover a segurança alimentar e nutricional da população brasileira.

\subsection{Operacionalização e Possíveis Impactos do PAA no Contexto da COVID-19}

Pesquisadores, movimentos sociais e representantes da agricultura familiar têm defendido que, diante dos resultados positivos produzidos pelo PAA ao longo de sua existência, o programa pode assumir um papel relevante e estratégico no enfrentamento aos desafios da pandemia provocada pelo novo coronavírus, tanto para a produção de alimentos quanto para o desenvolvimento da economia local (ANA, 2020).

Como apresentado neste artigo, o Brasil lida com um alto número de pessoas que vivem em situação de fome e miséria. De acordo com a FAO (2020b), esse número pode aumentar em decorrência da pandemia. Por isso, há a recomendação de construir medidas que evitem interrupções da cadeia de suprimentos de alimentos. No país, o desafio maior é manter o abastecimento, pois, além das dificuldades com logística e comercialização provocadas pelas restrições sanitárias impostas pela COVID-19, há também uma série de problemas conjunturais preexistentes, como a crise econômica e o processo de desconstrução de "estruturas de proteção e de estímulo à produção agrícola familiar" que vêm ocorrendo nos últimos anos (Valadares et al., 2020).

Por isto o PAA é tão importante em âmbito econômico e social: se por um lado escoa os produtos da agricultura familiar, por outro combate a fome e a miséria de famílias que vivem em situação de vulnerabilidade. Ademais, atua como ponte na comunicação entre o rural e o urbano, já que constrói em torno da política toda uma rede baseada na reciprocidade. Assim, o PAA apresenta elementos estratégicos de resposta central e urgente contra o risco de desabastecimento e a garantia de renda e segurança alimentar no campo e nas cidades (Valadares et al., 2020).

Atores envolvidos na implementação do PAA apontam que é importante a retomada das compras do programa, em especial via compra direta, compra com doação simultânea e formação de estoque (ANA, 2020), dando também continuidade à modalidade PAA-Leite (Valadares et al., 2020). Assim, visa-se ao estímulo a circuitos curtos de comercialização - que podem fazer escoar rapidamente a produção -, à dinamização da economia, à doação de alimentos para equipamentos socioassistenciais, e, no caso da formação de estoque, à antecipação de recursos a organizações da agricultura familiar.

O PAA, entretanto, não pode ser analisado de modo isolado. Para que alcance resultados e tenha impacto positivo na economia e na estruturação de cadeias alimentares, faz-se necessário tratá-lo de modo intersetorial e relacionado a outras políticas públicas, como a de Segurança Alimentar e Nutricional (SAN), a de Assistência Técnica e Extensão Rural (Ater) e a de crédito.

Do ponto de vista da SAN, a orientação do Conselho Nacional de Saúde (CNS) "é ampliar a distribuição de alimentos e cestas emergenciais pelos equipamentos de segurança alimentar e nutricional (restaurantes populares, cozinhas comunitárias e bancos de alimentos), priorizando 
as organizações de assistência social que atendem aos grupos de risco, as organizações de longa permanência e as que podem apoiar diferentes redes locais de solidariedade" (CNS, 2020).

Uma recomendação importante é que essas medidas obedeçam às orientações para uma alimentação saudável. De acordo com o CNS (2020), a alimentação baseada no consumo de alimentos in natura e minimamente processados é uma forma de evitar infecções e outras doenças, garantindo a saúde da população e impedindo a sobrecarga do atendimento pelo Sistema Único de Saúde (SUS) diante da pandemia. Por esse motivo, o Conselho solicitou o retorno imediato do PAA, além da recomendação de reconstituir a Câmara Interministerial de Segurança Alimentar e Nutricional (Caisan) e reativar e fortalecer os Conselhos de Segurança Alimentar (Consea).

A indicação de reativação do PAA pelo CNS se baseia também no potencial do programa para promover a diversificação da dieta e incentivar hábitos alimentares saudáveis. Segundo estudo realizado por Sambuichi et al. (2019b), por exemplo, o PAA-CDS chegou a adquirir 536 itens diferentes no período de 2011 a 2018, com média de 345 itens ao ano. Entre os produtos, incluem-se diversas categorias de alimentos in natura, como frutas, hortaliças, legumes, raízes, ovos, carnes, cereais e mel, além de uma variedade de produtos da agroindústria familiar, como queijos, polpas, doces, farinhas, pães, bolos e conservas, observando-se também produtos da sociobiodiversidade, como açaí, castanha-do-brasil e azeite de babaçu, entre outros.

Atualmente, os serviços de Ater têm procurado se adequar à nova realidade, promovendo cursos e aulas on-line, divulgando orientações e cartilhas por meio de redes sociais e construindo canais de comunicação via aplicativos de celular. No entanto, as informações ficam restritas a um público específico - aqueles que têm acesso à internet e/ou estão próximos ao meio urbano -, tornando difícil o acesso por agricultores familiares que vivem em regiões mais remotas no interior do Brasil (Agência Nacional de Assistência Técnica e Extensâo Rural [Anater], 2020).

Além disso, faz-se necessária a retomada de investimentos em Ater no Brasil, já que desde $2015 \mathrm{o}$ orçamento de políticas voltadas ao desenvolvimento rural sustentável vem sendo reduzido de modo acentuado. Despesas com ações voltadas para o desenvolvimento da agricultura familiar, como o próprio PAA e ações de Ater para pequenos produtores, tiveram redução de 33,7\% a 99,9\% entre 2014 e 2018 (Fian Brasil, 2019).

Quanto à proteção financeira e produtiva da agricultura familiar, a prorrogação da quitação do crédito concedido aos agricultores - a exemplo do Programa Nacional de Fortalecimento da Agricultura Familiar (Pronaf) -, a criação de novas linhas de crédito emergencial, a disponibilização de recursos contra problemas climáticos e a antecipação de crédito para famílias assentadas são algumas das medidas que podem ser adotadas nesse sentido (Valadares et al., 2020).

Levando em conta a recomendação desta pesquisa de implementar o PAA em um cenário otimizado, alguns elementos conjunturais devem ser observados, como a criação de demanda, a viabilidade de produção, a flexibilização da burocracia e a adaptação à nova realidade para recebimento e distribuição de produtos.

Os dados desta pesquisa evidenciaram que, no momento atual, a demanda se concretiza principalmente pelo funcionamento da rede socioassistencial e de hospitais e equipamentos públicos de segurança alimentar e nutricional (Epsans). Restaurantes populares, bancos de alimentos, cozinhas comunitárias e unidades de distribuição da agricultura familiar são serviços essenciais cujo funcionamento, quando adequado às normas sanitárias de combate à pandemia, é fundamental para, além de garantir à população vulnerável o acesso aos alimentos, manter a demanda pelos produtos 
da agricultura familiar, evitando a deterioração dos itens perecíveis e o aumento do desperdício de alimentos nas unidades familiares rurais.

Por outro lado, é preciso analisar também se a agricultura familiar teria capacidade de produção suficiente para abastecimento dos Epsans, caso haja aumento dos recursos disponibilizados para as compras de alimentos por meio de políticas públicas como o PAA em decorrência da COVID-19. De acordo com informações levantadas nesta pesquisa, há uma alta demanda das organizações da agricultura familiar pelo programa, pois a situação no campo vem se agravando nos últimos anos e a pandemia tem intensificado problemas como os de acesso a mercados. A Conab realizou, nos últimos três anos, uma análise de correlação para validar os critérios de distribuição de recursos por estado e identificou uma alta correlação entre a distribuição de recursos e a demanda que chega pelos projetos das organizações de agricultores familiares, mostrando haver uma demanda reprimida por parte dos agricultores para acessar o programa, ou seja, eles têm produtos para oferecer se houver para quem vender.

Em relação à viabilidade da produção, um aspecto importante é a oferta de Ater. Para o bom funcionamento do PAA, ainda mais durante a crise sanitária, acredita-se que, com a assistência técnica especializada e adequada à sua realidade, os agricultores podem ser orientados quanto ao planejamento e organização da produção (garantindo eficiência e evitando perdas), ao armazenamento e transporte, bem como à comercialização - visando à prevenção do contágio. Além disso, esse pode ser um canal para auxiliar a remanejar a produção de acordo com as dinâmicas locais, direcionando o excedente para o consumo mediante técnicas de aproveitamento e preparo, mantendo a qualidade da alimentação da família (Valadares et al., 2020).

Do ponto de vista da diminuição da burocracia, o ideal seria simplificar os processos para a aquisição dos alimentos, dispensando os documentos que não forem estritamente necessários, em função do caráter emergencial. A ANA (2020) sugere que é possível que aspectos sanitários possam ser flexibilizados, desde que não incorram em riscos à saúde pública, e que a análise laboratorial dos alimentos possa ser realizada por amostragem, em vez de todos os lotes. Isso daria agilidade ao processo de compra e distribuição, como apontam também as organizações da sociedade civil.

Desse modo, de acordo com os dados deste estudo, pode-se perceber que existe um esforço para a simplificação do processo por parte dos executores, de modo que a parte operacional e financeira flua sem paralisações que comprometam o andamento da política em nível local. Alguns documentos que antes exigiam reuniões agora são dispensados, a fim de dar mais celeridade ao processo burocrático. A ideia dos executores é garantir as entregas da agricultura familiar e o controle de documentos sem que isso acarrete prejuízos e riscos de contaminação por alimentos aos beneficiários consumidores.

Quanto às adaptações necessárias ao recebimento e à distribuição dos alimentos, se inferiu dos dados coletados que há uma mobilização para viabilizar a implementação do PAA. Foram feitas orientações por meio de ofícios, desde o início da pandemia, no sentido de realizar agendamentos para a entrega de cestas, a fim de evitar aglomerações.

De acordo com os entrevistados, muitos estados do Brasil implementaram uma série de adaptações do ponto de vista sanitário, como é o caso de Rio Grande do Norte, Bahia, Piauí, Paraná e Minas Gerais, que definiram entregas dos alimentos em dias predeterminados, com agendamento em dias e horários diferentes para os agricultores familiares. A distribuição passou a ser descentralizada, evitando concentração de pessoas em um único local. Há também os casos em que as entregas são feitas diretamente nas entidades assistidas. Para realizar as atividades de recebimento e distribuição de 
alimentos, os agentes envolvidos têm feito uso de equipamentos de segurança como luvas descartáveis, touca, máscaras, álcool em gel, além de manter o distanciamento entre pessoas, como exigido pelos órgãos sanitários.

Os dados coletados apontam que esses esforços não têm se concentrado especificamente no PAA. Há também a adoção de protocolos com base nas orientações dadas pela OMS e pelo Ministério da Saúde tanto por parte do PNAE - nos locais em que este se encontra em funcionamento - quanto pelas feiras livres, quando permitidas.

As adaptações do PAA e das demais políticas de aquisição de alimentos, assim como as feiras livres, mostraram que há condições de operacionalizar programas públicos em meio à pandemia com a adoção de alguns cuidados. Embora protocolos e medidas de segurança não sejam uma realidade para todos os municípios e regiões do Brasil, podem ser replicados. Para isso, contudo, são necessários mobilização e planejamento logístico entre o governo central e o local, de modo que se padronizem minimamente as normas e as condutas a serem adotadas como medidas de segurança sanitária, visando ao bom funcionamento dos programas públicos de apoio à comercialização da agricultura familiar.

\section{CONCLUSÃO}

Ao longo de sua trajetória, o PAA mostrou ser uma ferramenta capaz de promover estruturação de cadeias produtivas, acesso a novos mercados e indução ao dinamismo econômico, além de vários benefícios na área social, como aumento do acesso à alimentação saudável e melhoria da saúde. $\mathrm{O}$ programa tem um importante papel na promoção da saúde da população mais vulnerável, promovendo acesso a alimentos saudáveis e melhorando a qualidade da alimentação de pessoas em situação de risco alimentar.

Da maneira como está estruturado, o programa vai ao encontro das recomendações da FAO e das demandas da sociedade civil e de movimentos sociais para combater os danos causados pela COVID-19, pois colabora para manter a cadeia alimentar viva, como precaução contra os impactos na oferta de alimentos que ocorrem neste momento e os impactos na demanda que podem ocorrer após a pandemia. Além disso, o fortalecimento do PAA como ferramenta de combate à crise é uma medida tempestiva, tendo em vista que o programa já está criado e consolidado, com estrutura de implementação ativa.

A análise quantitativa mostrou que houve uma tendência acentuada de redução do volume de recursos aplicados no PAA nos últimos anos, ao passo que as projeções indicaram que o valor orçado para 2020 reduziria ainda mais o número de beneficiários e a abrangência do programa. Por outro lado, o incremento de recursos, em especial em um cenário otimizado, demonstrou possibilitar uma ampla capilarização dos benefícios gerados, o que poderá proporcionar melhores condições de vida dos beneficiários e amenizar os impactos sociais que derivam das crises sanitária e econômica.

Para a retomada satisfatória do programa em um cenário otimizado, é necessário se pensar na criação de demanda, flexibilização da burocracia e adaptação à nova realidade para recebimento e distribuição de produtos. O levantamento qualitativo evidenciou que o PAA, por meio de executores e agentes envolvidos, tem se mostrado eficaz na construção de procedimentos, protocolos e arranjos locais, permitindo a operacionalização da política.

Destaca-se também a importância da coordenação e da transparência em sua implementação. $O$ controle social foi uma ferramenta essencial nesse aspecto, proporcionando, por meio da participação 
local da sociedade civil, a adequação e a fiscalização das ações. Desse modo, a reabertura de tais mecanismos contribui para a articulação entre governo e sociedade, estabelecendo o diálogo entre fornecedores e consumidores de alimentos, melhorando, assim, a adequação dos processos frente à crise e às demandas regionais.

Dessa forma, considerando as previsões nacionais e internacionais de agravamento da crise sanitária, o posterior abalo econômico e o potencial do PAA para combater os efeitos da crise, especialmente sobre a população mais vulnerável, este trabalho recomenda que sejam ampliadas as suas ações, com a destinação de mais recursos para o programa. Recomenda-se ainda que o Governo Federal, junto com esferas locais, construa, de modo unificado, documentos informativos com protocolos e procedimentos gerais a serem adotados durante a pandemia no que tange a recebimento, distribuição e exigências sanitárias. Ademais, além dos recursos disponibilizados durante a pandemia, sugere-se que sejam disponibilizados recursos adicionais, iguais ou superiores, nos anos subsequentes, a fim de minimizar os impactos do pós-pandemia, dando continuidade aos efeitos positivos do PAA no apoio à economia e na promoção da segurança alimentar e nutricional da população. 


\section{REFERÊNCIAS}

Agapto, J. P., Borsatto, R. S., Esquerdo, V. F. de S., \& Bergamasco, S. M. P. P. (2012). Avaliação do Programa de Aquisição de Alimentos (PAA) em Campina do Monte Alegre, Estado de São Paulo, a partir da percepção dos agricultores. Informações Econômicas, 42(2), 13-21.

Agência Nacional de Assistência Técnica e Extensâo Rural. (2020). Webinar sobre prevenção à covid-19 no meio rural reúne centenas de participantes. Recuperado de http://www.anater.org/

Agostini, C., \& Bourscheidt, H. J. (2018). A Implementação do Programa de Aquisição de Alimentos nos Municípios do Vale do Taquari (RS): uma análise da percepção dos executivos municipais. Revista de Economia e Sociologia Rural, 56(2), 276-292.

Almeida, A. F. C. S., Perin, G., Policarpo, M. A., \& Sambuichi, R. H. (2020). O Programa de Aquisição de Alimentos (PAA): um caso de Big Push Ambiental para a sustentabilidade no Brasil. In Comissão Econômica para a América Latina e o Caribe. Repositório de casos sobre o big push para a Sustentabilidade no Brasil. Brasília, DF: Autor.

Andrade, R. C., Jr. (2009). O Programa de Aquisição de Alimentos da agricultura familiar (PAA) no Planalto Norte do estado de Santa Catarina: o caso da Cooperativa Agropecuária Regional de Pequenos Produtores de Mafra (Cooarpa ). Cadernos Do Ceom, 22(30).

Articulação Nacional de Agroecologia. (2020). Programa de Aquisição de Alimentos: Comida Saudável para o Povo. Recuperado de https:// agroecologia.org.br

Barth-Teixeira, E., Santos, C. T. dos, Gregolin, M. R., \& Christoffoli, P. I. (2017). Análise da Operacionalização do Programa de Aquisição de Alimentos (PAA/Conab) no Território Cantuquiriguaçu-PR. Anais do Congresso da Sober, Santa Maria, RS, Brasil, 55.

Brasil. (2020). Campanha apoia produtores orgânicos durante pandemia. Recuperado de https://www. gov.br/

Chmiekewska, D., Souza, D., \& Lourete, A. A. (2010). O Programa de Aquisição de Alimentos da Agricultura
Familiar (PAA) e as práticas dos agricultores participantes orientadas ao mercado: Estudo de caso no Estado de Sergipe (Texto Para Discussão, no 1510). Brasília, DF: Ipea.

Confederação Nacional da Agricultura e Pecuária. (2020). Impacto do coronavírus. Brasília, DF: Boletim CNA.

Conselho Nacional de Populações Extrativistas. (2020). Recomendação no 034, de 7 de maio de 2020. Brasília, DF: Autor.

Fundação Banco do Brasil. (2020). Carinho à mesa: parceria entre Emater-Rio e Fundação Banco do Brasil garante produção de cestas agroecológicas. Recuperado de www.fbb.org.br

Fian Brasil. (2019). Informe Dhana 2019: autoritarismo, negação de direitos e fome. Brasília, DF: Organização pelo Direito Humano à Alimentação e à Nutrição Adequadas.

Greene, W. H. (2007). Least Squares. In W. H. Greene (Ed.), Econometrics analysis. (6 ed., pp. 66-90). New Jersey, NJ: Prentice Hall.

Instituto Conexões Sustentáveis. (2020). Plano de resposta ao Covid-19. Recuperado de https://www. conexsus.org

Martins, S. P. (2013). PAA Leite: contexto de implantação, desenvolvimento e possibilidades de aprimoramento. In M. E. Del Grossi; D. R. Kroeff (Orgs). PAA: 10 anos de Aquisição de Alimentos. Brasília, DF: Ministério do Desenvolvimento Social e Combate à Fome.

Oliveira, T. C.; Abranches, M. V.; Lana, R. M. (2020). (In)Segurança alimentar no contexto da pandemia por Sars-CoV-2. Espaço temático: Covid-19 contribuições da saúde coletiva, 36(4), 1-6.

Organização das Nações Unidas para Alimentação e Agricultura. (2019). The state of food security and nutrition in the world 2019: safeguardin against economic slowdowns and downturns. (Relatório Técnico/2019). Roma, Italia: Food and Algriculture Organization of the United Nations.

Organização das Nações Unidas para Alimentação e Agricultura. (2020a). Mitigating impacts of COVID-19 on food trade and markets. Food and 
Algriculture Organization of the United Nations. Recuperado de www.fao.org.

Organização das Nações Unidas para Alimentação e Agricultura. (2020b). Q\&A: Covid-19 pandemic impact on food and agriculture. Recuperado de www.fao.org

Pontes, N. (2020). Pandemia sufoca agricultura familiar no Brasil. Recuperado de https://p.dw.com/ $\mathrm{p} / 3 \mathrm{~d} 3 \mathrm{yv}$

Rambo, A. G., \& Denz, E. von. (2015). Circuitos curtos de comercialização fomentando Novos usos do território: considerações sobre o PNAE e as feiras livres. In Anais do $7^{\circ}$ Seminário Internacional sobre Desenvolvimento Regional no Brasil, Santa Cruz do Sul, RS.

Salgado, R. J. S. F., Dias, M. M., Silva, E. A., Santos, L. F., \& Cunha, W. A. (2015). Implementação do Programa de Aquisição de Alimentos (PAA): Percepção dos atores envolcidos em sua operacionalização. In Anais do $53^{\circ}$ Congresso da Sober, João Pessoa, PB.
Sambuichi, R. H. R., Almeida, A. F. C. S., Perin, G., \& Moura, I. F. (2019a). Acesso ao Programa de Aquisição de Alimentos (PAA) no Brasil e Regiões. In Anais do $57^{\circ}$ Congresso da Sober, Ilhéus, BA.

Sambuichi, R. H., Perin, G., Almeida, A. F. C. S., Alves, P. S. C., Araújo, D. G., Câmara, R. D., ... Januário, E. S. (2019b). Diversidade de Produtos Adquiridos pelo Programa de Aquisição de Alimentos no Brasil e Regiões. Boletim Regional, Urbano e Ambiental, (21), 109-116.

Santos, S. R. Q., Soares, N. S., \& Benavides, Z. A. C. (2015). Programa de Aquisição de Alimentos da agricultura familiar (PAA): o caso dos produtores de Ibicaraí-ba. Revista de Estudos Sociais, 17(33), 161-182.

Valadares, A. A.; Alves, F.; Galiza, M.; \& Silva, S. P. (2020). Agricultura familiar e abastecimento alimentar no contexto do covid-19: uma abordagem das ações públicas emergenciais (Nota Técnica nº 69). Brasília, DF: Ipea. 


\section{Regina Helena Rosa Sambuichi}

https://orcid.org/0000-0002-2020-7944

Doutora em Ecologia pela Universidade de Brasília (UnB); Pesquisadora na Diretoria de Estudos e Políticas Regionais, Urbanas e Ambientais (Dirur) do Instituto de Pesquisa Econômica Aplicada (Ipea).

E-mail: regina.sambuichi@ipea.gov.br

\section{Ana Flávia Cordeiro Souza de Almeida}

https://orcid.org/0000-0001-6015-9011

Mestre em Administração pela Universidade Federal de Goiás (UFG); Pesquisadora na Diretoria de Estudos e Políticas Regionais, Urbanas e Ambientais (Dirur) do Instituto de Pesquisa Econômica Aplicada (Ipea).

E-mail: ana.cordeiro@ipea.gov.br

\section{Gabriela Perin}

ID

https://orcid.org/0000-0002-5138-5946

Mestre em Políticas Públicas pela Universidade Federal do Rio Grande do Sul (UFRGS); Pesquisadora na Diretoria de Estudos e Políticas Regionais, Urbanas e Ambientais (Dirur) do Instituto de Pesquisa Econômica Aplicada (Ipea). E-mail: gabriela.perin@ipea.gov.br

\section{Paulo Asafe Campos Spínola}

https://orcid.org/0000-0002-5436-063X

Mestre em Ciência Política pela Universidade de Brasília (UnB); Pesquisador na Diretoria de Estudos e Políticas Regionais, Urbanas e Ambientais (Dirur) do Instituto de Pesquisa Econômica Aplicada (Ipea).

E-mail: paulo.spinola@ipea.gov.br

\section{Antônio Fernando Costa Pella}

https://orcid.org/0000-0001-9992-8682

Mestre em Economia pela Universidade Federal do Espírito Santo (UFES); Pesquisador na Diretoria de Estudos e Políticas Regionais, Urbanas e Ambientais (Dirur) do Instituto de Pesquisa Econômica Aplicada (Ipea). E-mail: antonio.pella@ipea.gov.br 